\title{
Biomarcadores sanguíneos de cavalos após ingestão de milho processado de diferentes formas ou farelo de algaroba
}

\author{
Blood biomarkers in horses after ingestion of different \\ types of processed corn or mesquite pod meal
}

Helena Emília Cavalcanti da Costa Cordeiro Manso ${ }^{[a]}$, Aline Emmanuelle Maria da Silva ${ }^{[b]}$, Tito Alves Santiago ${ }^{[c]}$, Telga Lucena

Alves Craveiro de Almeida ${ }^{[\mathrm{d}]}$, Sílvia Robles Reis Duarte ${ }^{[\mathrm{e}]}$, Hélio Cordeiro Manso Filho ${ }^{[\mathrm{f}]}$

[a] Núcleo de Pesquisa Equina (NPE), Departamento de Zootecnia (DZ), Universidade Federal Rural de Pernambuco (UFRPE), Recife, PE - Brasil, e-mail: hecosta@dz.ufrpe.br

[b] Núcleo de Pesquisa Equina (NPE), Departamento de Zootecnia (DZ), Universidade Federal Rural de Pernambuco (UFRPE), Recife, PE - Brasil, e-mail: alinemmanuelle@gmail.com

[c] Núcleo de Pesquisa Equina (NPE), Departamento de Zootecnia (DZ), Universidade Federal Rural de Pernambuco (UFRPE), Recife, PE - Brasil, e-mail: titosantiago@gmail.com

[d] Núcleo de Pesquisa Equina (NPE), Departamento de Zootecnia (DZ), Universidade Federal Rural de Pernambuco (UFRPE), Recife, PE - Brasil, e-mail: telgalucena@hotmail.com

[e] InVivo Nutrição e Saúde Animal, Descalvado, SP - Brasil, e-mail: silvia_robles@invivo-nsa.com.br

[f] Núcleo de Pesquisa Equina (NPE), Departamento de Zootecnia (DZ), Universidade Federal Rural de Pernambuco (UFRPE), Recife, PE - Brasil, e-mail: hmanso@dz.ufrpe.br

\section{Resumo}

Esse experimento objetivou determinar a variação de diferentes biomarcadores sanguíneos após a ingestão de milho, sob diferentes tipos de processamentos, e do farelo da vagem de algaroba (FVA) em equinos em mantença. Foi utilizado um fatorial de cinco animais com cinco tratamentos: milho triturado (M-TR), milho extrusado (M-EX), milho floculado (M-FL), milho umedecido (M-UM) e FVA. Após 15 dias de suplementação, amostras de sangue foram colhidas com animais em jejum e 0,5; 1; 2; 3; 4; 5 e 6 horas após a suplementação. Foram determinadas as concentrações de glicose [GLIC], proteína plasmática total [PPT], hematócrito, ureia [UREIA], creatinina [CREAT], colesterol total [COLES-T] e triglicérides [TRIG]. Os resultados foram submetidos à ANOVA $(\mathrm{P}<0,01)$ e ao teste Tukey, como teste post hoc $(\mathrm{P}<0,05)$. Ocorreram variações na fase da colheita de sangue, tratamento e na interação fase/tratamento para a [GLIC] e [PPT] $(\mathrm{P}<0,01)$. Os parâmetros [UREIA], [CREAT] e [COLES-T] foram significativos nos tratamentos e os [CREAT], [COLES-T] e [TRIG] para a interação fase/tratamento $(\mathrm{P}<0,01)$. 0 FVA apresentou maiores [GLIC] $(\sim 8,0 \mathrm{mml} / \mathrm{L})$ e PPT $(\sim 6,9 \mathrm{~g} /$ $\mathrm{dL})$ e o M-TR as mais baixas [GLIC] $(\sim 4,6 \mathrm{mmol} / \mathrm{L})$, [PPT] $(\sim 6,2 \mathrm{~g} / \mathrm{dL})$, [COLE-T] $(\sim 1,3 \mathrm{mmol} / \mathrm{L})$, [UREIA] $(\sim 3,5 \mathrm{mmol} / \mathrm{L})$ e [CREAT] $(\sim 57 \mu \mathrm{mol} / \mathrm{L})$. Ainda, FVA apresentou maiores [GLIC] no pico $(\sim 11 \mathrm{mmol} / \mathrm{L}) \mathrm{e}$ 6,0 horas $(\sim 6,7 \mathrm{mmol} / \mathrm{L})$, além de apresentar a maior área sob a curva $(\sim 58)$, diferentemente do M-TR, que apresentou menores máxima $(\sim 5,4 \mathrm{mmol} / \mathrm{L})$ e área sob a curva $(\sim 32)(\mathrm{P}<0,05)$. Conclui-se que o tipo de processamento modifica a concentração de alguns biomarcadores utilizados na nutrição equina e que o 
FVA é capaz de produzir variações mais elevadas quando comparado com o milho sob diferentes formas de processamento.

Palavras-chave: Glicose. Triglicérides. Ureia. Energia. Equino.

\section{Abstract}

The objective of the present study was to determine the variation occurring in several equine blood biomarkers after ingestion of mesquite pod bran (MPB) and corn, under different kinds of processing, on horses in maintenance. It was used a $5 \times 5$ factorial with five horses and five treatments: triturated corn (TC), extruded corn (EC), flaked corn (FC), wet corn (WC), and MPB. After 15 days of supplementation, blood samples were collected at the following times: fast and 0.5, 1, 2, 3, 4, 5 and 6 hours after supplementation. Concentrations of glucose [GLUC], total plasma protein [TPP], hematocrit, urea [UREA], creatinine [CREAT], total cholesterol [T-CHOLES], and triglycerides [TRIG] were determined. Results were submitted to ANOVA $(P<0.01)$, and Tukey Test as a post hoc test $(P<0.05)$. There were variations during times of blood collection, treatments, and in the interaction time/treatment for [GLUC] and [TPP] (P<0.01). The biomarkers [UREA], [CREAT], and [T-CHOLES] were significantly different in the treatments, and the biomarkers [CREAT], [T-CHOLES], and [TRIG] in the interaction time/treatment $(P<0.01)$. The MPB had higher [GLUC] $(\sim 8.0 \mathrm{mml} / \mathrm{L})$, and TPP $(\sim 6.9 \mathrm{~g} / \mathrm{dL})$. The TC had the lowest [GLUC] ( 4.6mmol/L), [TPP] ( 6.2g/dL), [T-CHOLES] ( 1.3mmol/L), [UREA] ( 3.5mmol/L), and [CREAT] $(\sim 57 \mu \mathrm{mol} / \mathrm{L})$. The highest peak [GLUC] $(\sim 11 \mathrm{mmol} / \mathrm{L})$ and $+6.0 \mathrm{hours}(\sim 6.7 \mathrm{mmol} / \mathrm{L})$, were seen for MPB, as well as the largest area under the curve ( 58), differently of TC, that had the lowest peak $(\sim 5.4 \mathrm{mmol} / \mathrm{L})$, and area under the curve $(\sim 32)(P<0.05)$. It is concluded that different kinds of processing modify the concentration of biomarkers used in equine clinical nutrition, and the MPB is capable of producing larger variations when compared to corn under different types of processing.

Keywords: Glucose. Triglycerides. Urea. Energy. Equine.

\section{Introdução}

Os metabólitos e constituintes do sangue passaram a ser referidos na literatura como biomarcadores, pois fornecem indicações sobre os processos biológicos, como por exemplo, as respostas aos desafios nutricionais. Nesse sentido, os biomarcadores podem ser mensurados e avaliados objetivamente, contribuindo para o entendimento das adaptações metabólicas frente aos desafios para a sobrevivência (Mamas et al., 2001). Diferentes estudos indicam que o processamento dos alimentos pode alterar os níveis de biomarcadores sanguíneos no período pós-prandial, facilitando o entendimento dos programas nutricionais e os possíveis efeitos sobre a digestão pré-cecal (Hill, 2007; NRC, 2007; Vervuert et al., 2004). Sendo assim, o melhor conhecimento dos efeitos dos alimentos processados sobre as possíveis variações nos biomarcadores sanguíneos pode ser importante para favorecer a saúde dos cavalos e o seu bem-estar.
A inclusão de alternativas alimentares, ou antigos alimentos submetidos aos novos métodos de industrialização, busca a redução do custo dos programas nutricionais nas criações de cavalos. No Nordeste, há duas práticas que ainda são pouco avaliadas no que diz respeito aos efeitos sobre os biomarcadores metabólicos. A primeira delas é o uso da vagem da algarobeira (Prosopis juliflora) in natura ou triturada, como descrito por Stein et al. (2005). A segunda delas implica em colocar o milho inteiro imerso em água por 8 a 12 horas, remover a água e lavar o produto para, em seguida, fornecê-lo aos equídeos. Em ambos os casos, não são conhecidos os efeitos sobre a concentração de alguns biomarcadores presentes no sangue dos animais, principalmente quanto à glicose, após a utilização dessas práticas. Diferentemente do que ocorre com as práticas citadas anteriormente, já há bastante informação sobre o que ocorre após os processos de floculação e extrusamento do milho (Vervuert et al., 2004; Hill, 2007). 
Modificações nas concentrações plasmáticas de biomarcadores, tanto do metabolismo intermediário como dos produtos finais, podem ser importantes indicativos das respostas às dietas que são oferecidas aos animais (Manso et al., 2015). Com o intuito de se conhecer os efeitos de alguns alimentos, regularmente oferecidos aos equinos no Nordeste, sobre as variações na concentração de biomarcadores sanguíneos, foi desenvolvido um estudo com o objetivo de determinar os efeitos da suplementação com milho, submetido a diferentes tipos de processamentos, e com farelo da vagem de algaroba sobre os biomarcadores sanguíneos ligados à nutrição clínica equina. 0 conhecimento das adaptações metabólicas por meio dos biomarcadores sanguíneos, após a ingestão dos alimentos descritos acima, poderá contribuir para a utilização deles com eficiência e economia.

\section{Material e métodos}

\section{Animais}

Foram utilizadas cinco éguas da raça PuroSangue Árabe, vazias, em mantença e alojadas na Universidade Federal Rural de Pernambuco (UFRPE), em Recife (PE). Esses animais foram pesados em balança eletrônica e a espessura da camada de gordura foi feita por ultrassonografia, para determinação da composição corporal (Manso Filho et al., 2009), antes e após as etapas experimentais. Os métodos empregados foram aprovados pelo Comitê de Ética e Bem-estar da UFRPE (\# 62/2007-CTA/DZ/UFRPE).

\section{Desenho experimental e programa de arraçoamento}

Os animais foram distribuídos aleatoriamente em fatorial de 5 animais com 5 tratamentos: milho triturado (M-TR), milho extrusado (M-EX), milho floculado (M-FL), milho inteiro umedecido em água potável por 12 horas (M-UM); e farelo da vagem de algaroba (FVA). A quantidade de alimentos no M-UM foi definida antes da adição da água, para depois ser fornecido aos animais. Após a definição da distribuição dos animais no desenho experimental, eles passavam por um período de 14 dias de adaptação ao tratamento. 0 concentrado, fornecido individualmente, correspondia a $50 \%$ da energia digestível $(\sim 3,5 \mathrm{Mcal} /$ animal/dia $)$ para animais em mantença e com peso médio de $400 \mathrm{Kg}$ (NRC, 2007). Todos os animais recebiam também feno de Tifton-85 (Cynodon spp.), à vontade, e tinham livre acesso aos piquetes com pastagem nativa. Água e sal mineralizado foram fornecidos ad libitum. Ao final de cada etapa experimental, os animais passavam por 21 dias de "wash-out", permanecendo nos piquetes sem receber concentrados, mas suplementados com o feno de Tifton-85.

\section{Colheita de sangue e processamento}

Amostras de sangue foram colhidas da veia jugular, em tubos a vácuo heparinizados, previamente resfriados, em oito ocasiões: animais em jejum e após 0,$5 ; 1 ; 2 ; 3 ; 4 ; 5$ e 6 horas da suplementação. Durante o período de colheita de sangue, os animais ficaram livres no curral e com acesso apenas à água. Imediatamente após a colheita de sangue, uma alíquota da amostra foi centrifugada para extração do plasma e outra refrigerada para determinação do hematócrito. 0 plasma obtido a partir da primeira alíquota foi novamente dividido em duas alíquotas, sendo uma imediatamente utilizada para determinação da glicose e proteína plasmática total, e a outra congelada a $-20{ }^{\circ} \mathrm{C}$, para determinação da ureia, creatinina, colesterol total e triglicérides. A concentração de glicose plasmática ([GLIC]) foi determinada em aparelho portátil (Accu-Check Advantage II, Roche $囚$ ) e a concentração das proteínas plasmáticas totais ([PPT]) através da refratometria. O hematócrito (HT) foi determinado pela técnica do micro-hematócrito. As determinações das concentrações de Ureia ([UREIA]), Creatinina ([CREAT]), Colesterol total ([COLE-T]) e Triglicérides ([TRIG]) foram realizadas em duplicata em equipamento semiautomático (DOLES $500 \AA$, Doles) e com kits comerciais. A área sob a curva (ASC) da glicose foi calculada conforme a literatura (Harris e Geor, 2009; Hoffman, 2009).

\section{Análise estatística}

Os resultados foram submetidos à análise da variância (ANOVA), para medidas repetidas e com 
um (tratamentos) ou dois fatores (tratamentos e as fases experimentais), com $\mathrm{P}<0,01$. 0 teste post hoc foi o teste de Tukey, com $\mathrm{P}<0,05$. 0 programa SigmaStat $₫ 13.0$ para Windows $₫$ foi utilizado para todas as análises estatísticas.

\section{Resultados}

Os resultados demonstram que apenas [GLIC] e [PPT] apresentaram variações nas fases da colheita, entre os tratamentos e na interação entre fases e tratamentos $(\mathrm{P}<0,01)$. Os demais biomarcadores não apresentaram variações na fase da colheita de sangue $(P>0,01)$, mas apresentaram variações entre tratamento para [CREAT], [UREIA] e [COLE-T] $(\mathrm{P}<0,01)$. Ocorreram ainda interações significativas entre as fases e os tratamentos para as [CREAT], [COLE-T] e [TRIG] $(\mathrm{P}<0,01)$. A percentagem do hematócrito não variou ao longo da colheita de sangue $(\mathrm{P}>0,01)$.

As comparações entre os tratamentos, pelo ANOVA com dois fatores, demonstraram que o tratamento FVA apresentou maiores médias para a [GLIC], [PPT], [COLE-T] e [CREAT] quando comparado com os demais tratamentos com milho sob diferentes tipos de processamentos $(\mathrm{P}<0,05)$ (Tabela 1$)$. A [UREIA] foi maior no M-EX e o M-TR apresentou as menores médias em todos os biomarcadores, inclusive a [GLIC] (Tabela 1 ).

Os resultados também foram analisados comparando-se as [GLIC] entre os tratamentos em períodos com significado metabólico (jejum, concentração máxima e após 6 horas), e na área sob a curva (ASC) da glicose, pelo ANOVA "one-way". Neles, foi observado que o tratamento FVA produziu os maiores valores da glicose para a concentração máxima e para a concentração após 6 horas $(\mathrm{P}<0,05)$, e nesse tratamento também foi observada a maior ASC da glicose $(\mathrm{P}<0,05)$ (Tabela 2). Não ocorreram diferenças na [GLIC] com os animais em jejum $(\mathrm{P}<0,05)$ (Tabela 2$)$.

Finalmente, foi observado que, em todas as etapas da experimentação, os alimentos foram consumidos em menos de 30 minutos e não ocorreram modificações na massa corporal e na percentagem de gordura. A massa corporal média dos animais foi de $\sim 380,0 \mathrm{Kg}$ e a percentagem de gordura de $\sim 11,5 \%$. Os resultados das análises bromatológicas dos alimentos estão na Tabela 3.

Tabela 1 - Resultado das comparações entre os tratamentos dos biomarcadores sanguíneos, em equinos, após a suplementação com milho, sob diferentes tipos de processamentos, e com farelo da vagem de algaroba

\begin{tabular}{|c|c|c|c|c|c|}
\hline \multirow{2}{*}{ Biomarcador } & \multicolumn{5}{|c|}{ Tratamento } \\
\hline & M-TR & M-EX & M-FL & M-UM & FVA \\
\hline $\begin{array}{l}\text { Glicose } \\
\text { (mmol/L) }\end{array}$ & $4,55+0,38^{d}$ & $6,95+0,38^{\mathrm{ab}}$ & $6,45+0,38^{\mathrm{abc}}$ & $5,52+0,38^{\text {bcd }}$ & $8,00+0,38^{a}$ \\
\hline $\begin{array}{l}\text { Proteína plasmática total } \\
\qquad(\mathrm{g} / \mathrm{dL})\end{array}$ & $6,17+0,09^{b}$ & $6,25+0,09^{b}$ & $6,28+0,09^{b}$ & $6.72+0,09^{a}$ & $6,87+0,09^{a}$ \\
\hline $\begin{array}{l}\text { Hematócrito } \\
(\%)\end{array}$ & $26,47+0,98$ & $26,84+0,98$ & $25,62+0,98$ & $30,09+0,98$ & $27,09+0,98$ \\
\hline $\begin{array}{l}\text { Ureia } \\
(\mu \mathrm{mol} / \mathrm{L})\end{array}$ & $3,51+0,40^{c}$ & $6,85+0,40^{a}$ & $4,76+0,40^{b c}$ & $5,21+0,40^{\mathrm{abc}}$ & $5,30+0,40^{\mathrm{ab}}$ \\
\hline $\begin{array}{l}\text { Creatinina } \\
(\mathrm{mmol} / \mathrm{L})\end{array}$ & $56,91+3,93^{c}$ & $82,05+3,93^{b}$ & $66,03+3,93^{b c}$ & $107,85+3,9^{\mathrm{a}}$ & $116,50+3,9^{a}$ \\
\hline $\begin{array}{l}\text { Colesterol Total } \\
\text { (mmol/L) }\end{array}$ & $1,26+0,10^{b}$ & $1,40+0,10^{b}$ & $1,31+0,10^{b}$ & $2,39+0,10^{\mathrm{a}}$ & $1,99+0,10^{\mathrm{a}}$ \\
\hline $\begin{array}{l}\text { Triglicérideos } \\
\text { (mmol/L) }\end{array}$ & $0,243+0,037$ & $0,304+0,037$ & $0,313+0,037$ & $0,185+0,037$ & $0,317+0,037$ \\
\hline
\end{tabular}

Legenda: $\mathrm{M}-\mathrm{TR}=$ milho triturado; $\mathrm{M}-\mathrm{EX}=$ milho extrusado; $\mathrm{M}-\mathrm{FL}=$ milho floculado; $\mathrm{M}-\mathrm{UM}=$ milho umedecido; $\mathrm{FVA}=$ farelo da vagem de algaroba. Nota: Diferentes letras na mesma linha indicam $P<0,05$ pelo teste de Tukey. 
Tabela 2 - Concentração da Glicose (mmol/L) (jejum, máxima e +6 horas) e a área sob a curva de diferentes tratamentos em equinos em mantença, após a suplementação do milho, sob diferentes tipos de processamentos, e com farelo da vagem de algaroba

\begin{tabular}{|c|c|c|c|c|}
\hline \multirow{2}{*}{ Tratamento } & \multicolumn{3}{|c|}{ [Glicose], mmol/L } & \multirow{2}{*}{ Área sob a curva da glicose } \\
\hline & Jejum & Máxima & Após 6 horas & \\
\hline M-Triturado & $4,42 \pm 0,10$ & $5,45 \pm 0,27^{b}$ & $4,23 \pm 0,21^{\mathrm{cd}}$ & $32,11 \pm 0,69^{d}$ \\
\hline M-Extrusado & $4,05 \pm 0,09$ & $10,25 \pm 1,25^{a}$ & $4,90 \pm 0,14^{b c}$ & $51,23 \pm 4,64^{\mathrm{ab}}$ \\
\hline M-Floculado & $3,82 \pm 0,09$ & $10,70 \pm 0,83^{\mathrm{a}}$ & $3,12 \pm 0,60^{d}$ & $48,10 \pm 2,92^{b c}$ \\
\hline M-Umedecido & $4,17 \pm 0,14$ & $7,22 \pm 0,90^{b}$ & $5,77 \pm 0,36^{\mathrm{ab}}$ & $39,38 \pm 3,53^{\mathrm{cd}}$ \\
\hline FVA & $4,55 \pm 0,20$ & $11,05 \pm 0,46^{\mathrm{a}}$ & $6,77 \pm 0,45^{\mathrm{a}}$ & $58,34 \pm 1,63^{\mathrm{a}}$ \\
\hline
\end{tabular}

Legenda: $\mathrm{M}=$ milho; $\mathrm{FVA}=$ farelo da vagem de algaroba.

Nota: Diferentes letras na mesma coluna indicam que $\mathrm{P}<0,05$ pelo teste de Tukey.

Tabela 3 - Análise bromatológica dos alimentos fornecidos aos animais

\begin{tabular}{|c|c|c|c|c|c|c|}
\hline \multirow{2}{*}{ Alimento } & \multicolumn{6}{|c|}{ Resultados da Análise Bromatológica } \\
\hline & MS, \% & $\mathrm{PB}, \%$ & $\mathrm{EE}, \%$ & $\mathrm{MM}, \%$ & $\mathrm{FDN}, \%$ & CNE \\
\hline M-Triturado & 89,20 & 11,70 & 8,95 & 2,50 & 27,36 & 49,49 \\
\hline M-Extrusado & 88,72 & 7,39 & 3,51 & 0,82 & 38,16 & 50,12 \\
\hline M-Floculado & 91,89 & 10,50 & 8,90 & 2,11 & 21,00 & 57,49 \\
\hline M-Umedecido & 86,71 & 5,43 & 2,85 & 0,76 & 35,60 & 55,36 \\
\hline FVA & 96,19 & 9,31 & 1,30 & 3,63 & 25,12 & 60,64 \\
\hline Feno Tifton-85 & 90,88 & 8,65 & 2,38 & 7,15 & 84,42 & ------ \\
\hline
\end{tabular}

Legenda: $\mathrm{MS}=$ matéria seca; $\mathrm{PB}$ = proteína bruta; $\mathrm{EE}$ = extrato etéreo; $\mathrm{MM}$ = matéria mineral; $\mathrm{FDN}$ = fibra em detergente neutro; $\mathrm{CNE}$ = carboidratos não estruturais; CNE= 100 - (PB\%+FDN\%+Umidade\%+EE\%+MM\%); FVA = farelo vagem de algaroba. 


\section{Discussão}

O estudo das variações na concentração dos biomarcadores sanguíneos no período pós-prandial pode ser importante para o entendimento do metabolismo nutricional nos equinos. Diferentes pesquisas têm demonstrado que o conhecimento dessas alterações pode ser importante para o conhecimento dos efeitos dos alimentos sobre o metabolismo geral dos cavalos (Ralston, 2002; Jose-Cunilleras et al., 2004; Harris e Geor, 2009). No atual experimento, apenas as [GLIC] e [PPT] apresentaram variações significativas nas fases da colheita de sangue e entre os tratamentos, indicando que esses biomarcadores podem ter modificações agudas no período pós-prandial, em contraste com as [UREIA], [CREAT], [COLE-T] e [TRIG], que parecem possuir uma variação mais crônica e associada à adaptação ao programa alimentar. Finalmente, deve-se observar que o hematócrito não modificou ao longo das fases de colheita de sangue, mesmo havendo uma modificação na [PPT], podendo indicar que a alteração no volume plasmático não foi elevada e, com isso, pouco influenciou as alterações observadas nos diferentes biomarcadores sanguíneos analisados.

Os resultados obtidos com o milho, submetido a diferentes processamentos, produziu curvas da glicose semelhantes às encontradas em outros estudos, com picos entre 2 e 3 horas após a suplementação (Vervuert et al., 2004; Harris e Geor, 2009). Ralston (2002), estudando a curva da glicose após testes com glicose oral e com grãos, observou que podem ocorrer diferenças no momento do pico da [GLIC] dependendo do alimento. Deve-se recordar que os valores individuais desse biomarcador do metabolismo dos carboidratos podem não ser similares entre diferentes experimentos e testes de desafio à glicose, por motivos como composição do alimento, velocidade de consumo, esvaziamento gástrico, digestão de carboidratos não-estruturais, quantidade de alimento fornecido, uso de medicamentos e fisiologia individual dos animais (Ralston, 2008; Vervuert et al., 2009; Harris e Geor, 2009; Mélo et al., 2014), o que dificulta as comparações entre as pesquisas, mas servem para melhorar o entendimento sobre esse processo.

O milho triturado, pouco contribuiu para melhorar a digestão pré-cecal. Diferentes pesquisadores afirmam que uma baixa resposta glicêmica de um alimento rico em carboidratos não-estruturais pode produzir efeitos adversos no colo e ceco dos equinos, como são os casos do milho inteiro e do milho triturado (Ralston, 2008; Harris e Geor, 2009; Hoffman, 2009). Em contrapartida, o processamento da extrusão e floculação melhora significativamente a digestibilidade do milho, o que pode ser comprovado pela grande variação na [GLIC] após a ingestão do M-EX e do M-FL. Esse fato é facilmente identificado quando analisa-se o comportamento das áreas sob as curvas (ASC) desse biomarcador para os diferentes tratamentos, com maiores ASC no FVA e no M-EX, seguido por M-FL, e que foram diferentes do M-TR $(\mathrm{P}<0,05)$. Não foi observada diferença na [GLIC] máxima e na ASC entre o M-UM e M-TR $(\mathrm{P}>0,05)$.

Neste experimento, observou-se que os animais em jejum apresentaram [GLIC] dentro da normalidade e sem diferenças significativas entre os tratamentos, todavia foram observadas diferenças na [GLIC] máxima, que ocorreu entre 120 e 180 minutos do período pós-prandial, como era esperado, pois diferentes autores indicam que o pico da glicose ocorre nesse período (Ralston, 2002; Myers et al., 2009). Quase todas [GLIC] máximas foram semelhantes às descritas na literatura $(6,7-9,9 \mathrm{mmol} / \mathrm{L})$ (Ralston, 2002; Meló et al., 2014), com a exceção do tratamento M-TR $(\sim 5,4 \mathrm{mmol} / \mathrm{L})$, indicando que ocorreu uma reduzida digestão pré-cecal do M-TR no período pós-prandial. Perry (2009) e Mélo et al. (2014) observaram que a [GLIC] após 12 horas de jejum pode variar de 4,0 a 7,0 $\mathrm{mmol} / \mathrm{L}$ nos cavalos, sendo que outros autores consideram que há hiperglicemia quando a [GLIC] passa de $\sim 7,0 \mathrm{mmol} / \mathrm{L}$ nessa fase (Ralston, 2002; Myers et al., 2009). Ao final das 6 horas de avaliação experimental, as [GLIC] reduziram-se e ficaram abaixo de 7,0 $\mathrm{mmol} / \mathrm{L}$, entretanto, para o FVA, a [GLIC] $(\sim 6,8$ $\mathrm{mmol} / \mathrm{L}$ ) ficou próximo ao valor para a hiperglicemia. Finalmente, Ralston (2002) comenta que, durante o teste de desafio à glicose, quando a [GLIC] passa dos 14,0 mmol/L, deve-se considerar que ocorreu uma resposta anormal e o procedimento deve ser melhor investigado, o que não foi observado neste experimento para a quantidade de alimento utilizado.

Outro alimento que vem ganhando importância na alimentação dos equídeos na região Nordeste é a vagem da algaroba (Stein et al., 2005), seja ela fornecida como fonte alternativa de energia ou como palatabilizante. No atual estudo, o farelo da vage de algaroba (FVA) foi o que produziu variações mais elevadas na [GLIC]. No estudo de Stein et al. (2005), foi demonstrado que o FVA pode ser utilizado como substituto 
do milho desintegrado com palha e sabugo sem afetar o consumo de energia digestível em equinos em mantença. O FVA produziu o maior pico da [GLIC] ( 11mmol/L) e também a maior concentração após 6 horas do desafio $(\sim 6,8 \mathrm{mmol} / \mathrm{L})$, próximo ao teto da normalidade para os equinos $(\sim 4,0-7,0 \mathrm{mmol} / \mathrm{L})$ (Ralston, 2002; Perry, 2009). Ainda constatou-se que a [GLIC], na fase +6 horas do tratamento FVA, foi quase $50 \%$ superior ao observado no mesmo tratamento com os animais em jejum.

A avaliação de um alimento energético rico em carboidratos não-estruturais pode ser realizada tanto pela análise da área sob a curva da glicose (ASC) como pelo índice glicêmico do alimento (Ralston, 2002; Harris e Geor, 2009). Alimentos ricos em carboidratos podem produzir grandes ASC (Hoffman, 2009) e, com isso, diferentes efeitos no metabolismo energético dos cavalos. Neste experimento, os tratamentos FVA $(\sim 58)$ e M-EX ( 51$)$ apresentaram maiores ASC, seguidos por M-FL ( $\sim 48)$, o que era esperado, pois o FVA apresentou maior percentagem de CNE. Já para o cálculo do índice glicêmico, foi utilizado o tratamento M-TR como padrão $(100 \%)$ para os cálculos dos índices glicêmicos dos demais alimentos, e foi constatado que os tratamentos M-EX, M-FL e FVA apresentaram índices glicêmicos de 60\%,50\% e 80\%, respectivamente, superiores ao padrão M-TR, enquanto que o M-UM foi apenas $20 \%$ superior.

Grandes elevações no índice glicêmico ou na ASC podem ter implicações sobre a saúde dos cavalos quando estes são suplementados com quantidades elevadas de concentrados que tem elevado índice glicêmico ou ASC ou quando os mesmos são utilizados como única fonte de energia. Diferentes pesquisadores demonstraram que alimentos muito ricos em carboidratos não-estruturais ou que produzam modificações exageradas nas [GLIC], e com isso nas de insulina, podem estar associados à enfermidades tão variadas, como osteocondrose, em animais jovens, ou laminite, nos adultos (Ralston, 2002; Harris e Geor, 2009; Hoffman, 2009). Em contraste, o fornecimento de alimentos ricos em carboidratos não-estruturais, mas que produzam baixos índices glicêmicos, ou seja, com baixa digestão pré-cecal, poderiam provocar enfermidades no trato digestório dos equinos ao modificar o padrão de fermentação e a microbiota no intestino grosso (Vervuert et al., 2004; Harris e Geor, 2009; Vervuert et al., 2009). Desta forma, durante a suplementação com concentrados ricos em carboidratos não-estruturais e que produzam ou não elevado índice glicêmico, os técnicos devem observar essas características dos alimentos, assim como as necessidades energéticas dos animais, evitando tanto a sub como a sobre-alimentação.

As avaliações da [PPT], [UREIA] e [CREAT] podem ser importantes biomarcadores para avaliação do metabolismo das proteínas, também servindo como indicadores do status proteico nos equinos em diferentes condições (Kohn et al., 2005). Esses biomarcadores podem ainda ser empregados como parâmetro clínico para ajuste de dietas ou para identificar problemas de saúde ou no programa nutricional. Ao longo do experimento, observou-se que a [PPT] variou significativamente na fase, no tratamento e na interação. Avaliando-se a [PPT] conforme a fase, que apresentou variação altamente significativa, observou-se que a concentração mais elevada da PPT ocorreu quando os animais estavam na fase de jejum $(\sim 6,8 \mathrm{~g} / \mathrm{dL})$ e a mais baixa após 3 horas de ingestão dos alimentos $(\sim 6,3 \mathrm{~g} /$ dL).

Essa variação da [PPT] pode ser atribuída a uma adaptação do volume plasmático durante a fase de colheita de sangue, já que os animais tiveram livre acesso a água, mas passaram por um longo período sem ingerir alimentos. Deve-se ressaltar que as variações encontradas na [PPT], em todos os tratamentos, estavam dentro dos valores da normalidade (Perry, 2009), indicando que as dietas foram capazes de manter as reservas proteicas no sangue dos animais. Finalmente, deve-se recordar que tratamentos não possuíam concentração elevada de proteína bruta mas eles não foram isoproteicos.

As concentrações de Ureia e a Creatinina podem estar elevados em equinos quando esses estão sendo suplementados com concentrados com proteína digestível elevada ou quando são suplementados com aminoácidos. Em equinos suplementados com uma mistura de Glutamina e Glutamato, durante 28 dias, observou-se elevação na [UREIA] e [CREAT], que passaram de $\sim 3,0$ para $\sim 4,0 \mathrm{mmol} / \mathrm{L}$ e de $\sim 90,2$ para $\sim 106,1 \mu \mathrm{mol} / \mathrm{L}$, respectivamente (Manso et al., 2015). Em contraste, animais medicados com diferentes doses de omeprazol, que modifica o pH gástrico e assim a digestão dos alimentos, apresentaram redução na [UREIA] e não nas de [PPT] e [CREAT] (Mélo et al., 2014). Esses estudos demonstram que esses biomarcadores são sensíveis à modificações tanto na quantidade de proteína na ração como nas modificações 
no processo digestivo. No atual experimento, a quantidade de proteína nos concentrados não era elevada $(<11 \%$ PB), mas, ainda assim, foi observado uma variação significativa entre os tratamentos nesses biomarcadores. Por fim, deve-se observar que, no atual experimento, os valores da [UREIA] e [CREAT] estão dentro da variação normal sugerida por Perry (2009) e aos descritos por Mélo et al. (2014) para cavalos em jejum.

0 hematócrito, que pode ser um bom indicador do nível de hidratação dos equinos, não se modificou na fase, tratamento, ou interação na atual pesquisa $(P>0,01)$, mesmo com a ocorrência de alterações significativas na [PPT]. A ausência de modificação na percentagem do hematócrito ao longo da fase de colheita de sangue era esperado, pois os animais se mantiveram calmos e não foram submetidos a fatores que pudessem estimular a contração esplênica, principal responsável por elevações rápidas desse biomarcador no sangue dos cavalos, e tinham livre acesso a água. As médias do hematócrito estavam pouco abaixo dos valores normais citados por Perry (2009), mas mesmo assim os animais não apresentaram sinais clínicos de anemia ou outra enfermidade. Em suma, deve-se enfatizar que, nos experimentos para se determinar o desafio à glicose, pode não haver mensuração do hematócrito, mas essas medidas, quando combinadas com a [PPT] são um bom indicativo do nível de hidratação do animal, como visto em experimentos com animais atletas (McKeever et al., 2006).

Os biomarcadores COLE-T e TRIG são largamente utilizados em análises de adaptações metabólicas à incorporação de óleos nos concentrados para equinos (Oldruitenborgh-Oosterbaan et al., 2002). No atual experimento, não ocorreram variações significativas nas fases de colheita de sangue para [COLE-T] e para [TRIG] ( $P>0,01)$, mas com diferenças entre os tratamentos $(\mathrm{P}<0,01)$. Esses resultados eram esperados, pois os alimentos utilizados não são fontes significativas de gordura para os cavalos. Também observou-se que os valores encontrados para esses biomarcadores estavam dentro de variações esperadas para cavalos sadios (Perry, 2009), todavia há indicação de que os equinos precisam de um longo período de adaptação aos concentrados com extrato etéreo para que surjam modificações nesses marcadores bioquímicos (Oldruitenborgh-Oosterbaan et al., 2002; NRC, 2007). Na atual pesquisa, os equinos foram tratados por apenas 14 dias e tinham um período de descanso entre as etapas da experimentação e, por essa razão, não era esperado variações nesses biomarcadores. Recentemente, Mélo et al. (2014) demonstraram que o tratamento com um redutor do $\mathrm{pH}$ (omeprazol) eleva a concentração desses biomarcadores no sangue de equinos, evidenciando que pode haver diferentes tipos de interação entre a ração e os produtos que atuem no processo digestivo dos equinos. Observando os valores da [COLE-T] no atual experimento, constata-se que eles são similares aos descritos por diferentes autores (Oldruitenborgh-Oosterbaan et al., 2002), mesmo com os animais ingerindo alimentos que apresentavam percentagem de extrato etéreo abaixo do utilizado por esses autores.

\section{Conclusões}

Conclui-se que equinos suplementados com alimentos processados, como milho e a vagem de algaroba, produzem modificações importantes na concentração dos biomarcadores da nutrição clínica, principalmente os ligados ao metabolismo de carboidratos. A extrusão e o floculamento do milho melhoram a digestão précecal desse alimento, quando comparados com o umedecimento e com a trituração. O FVA produziu maior variação da concentração da glicose e dos outros parâmetros ao ser comparado com os diferentes tipos de processamentos do milho, produzindo maiores desafios metabólicos para os equinos.

\section{Referências}

Harris P, Geor RJ. Primer on dietary carbohydrate and utility of the glycemic index in equine nutrition. Veterinary Clinics of North America: Equine Practice. 2009; 25(1):2337. doi:10.1016/j.cveq.2009.01.006.

Hill J. Impacts of nutritional technology on feeds offered to horses: a review of effects of processing on voluntary intake, digesta characteristics and feed utilisation. Animal Feed Science and Technology. 2007; 138(2):92-117. doi:10.1016/j.anifeedsci.2007.06.018.

Hoffman, RM. Carbohydrate metabolism and metabolic disorders in horses. Revista Brasileira de Zootecnia. 2009; 38:270-276. doi:10.1590/S1516-35982009001300027. 
Jose-Cunilleras E, Taylor LE, Hinchcliff KW. Glycemic index of cracked corn, oat groats and rolled barley in horses. Journal of Animal Science. 2004; 82:2623-2629.

Kohn RA, Dinneen MM, Russek-Cohen E. Using blood urea nitrogen to predict nitrogen excretion and efficiency of nitrogen utilization in cattle, sheep, goats, horses, pigs, and rats. Journal of Animal Science. 2005; 83(4):879-889.

Mamas M, Dunn WB, Neyses L, Goodacre R. The role of metabolites and metabolomics in clinically applicable biomarkers of disease. Archives of Toxicology. 2011; 85(1):517. doi:10.1007/s00204-010-0609-6.

Manso HECCC, Silva CJFL, Barbosa BL, Vasconcelos JLA, Manso Filho HE. Efeitos da suplementação com Glutamina e Glutamato sobre os índices hematimétricos e biomarcadores sanguíneos de equinos. Acta Scientiae Veterinariae. 2015; 43:1-7.

Manso Filho HC, Manso HECC, Ferreira LMC, Santiago TA, Wanderley EK, Abreu JMG. Percentagem de gordura em cavalos criados em região tropical. Acta Scientiae Veterinariae. 2009; 37(3):239-243.

McKeever JM, McKeever KH, Albeirci JM, Gordon ME, Manso Filho HC. Effect of omeprazole on markers of performance in gastric ulcer-free Standardbred horses. Equine Veterinary Journal. 2006; 36:668-671. doi:10.1111/j.2042-3306.2006. tb05624.x.

Mélo SKM, Santiago TA, Duarte TL, Abreu JMG, Manso HECCC, Manso Filho HC. A proteon-pump inhibitor modifies the concentration of digestion biomarkers in healthy horses. Journal of Equine Veterinary Science, 2014; 34(1112): 1318-1323. doi:10.1016/j.jevs.2014.09.013.

Myers CJ, Magdesian KG, Kass PH, Madigan JE, Rhodes DM, Marks ST. Parenteral nutrition in neonatal foals: clinical description, complications and outcome in 53 foals (19952005). The Veterinary Journal. 2009; 181(2):137-144. doi:10.1016/j.tvjl.2008.02.024.

National Research Council - NRC. Nutrient requirements of horses. 6 ed. Washington, DC: National Academy of Sciences, 2007.

Oldruitenborgh-Oosterbaan MMS, Annee MP, Verdegaal EJMM, Lemmens AG, Beynen AC. Exercise- and metabolism-associated blood variables in Standardbreds fed either a low- or a high-fat diet. Equine Veterinary Journal. 2002; 34(S34):29-32. doi:10.1111/j.2042-3306.2002.tb05387.x.
Perry BW. Clinical pathology reference data. In: Robinson NE, Sprayberry KA. Current Therapy in Equine Medicine. 6 ed. Philadelphia; 2009. p. 956-980.

Ralston SL. Influence of management on equine digestion. Revista Brasileira de Zootecnia. 2008; 37:211-214.

Ralston SL. Insulin and glucose regulation. Veterinary Clinics of North America: Equine Practice. 2002; 18(2):295304. doi:10.1016/S0749-0739(02)00014-7.

Stein RBS, Toledo LRA, Almeida FQ, Arnaut AC, Patitucci LT, Soares Neto J, Costa VTM. Uso do farelo de vagem de Algaroba (Prosopis juliflora (Swartz) D.C.) em dietas para equinos. Revista Brasileira de Zootecnia. 2005; 34(4):12401247. doi:10.1590/S1516-35982005000400020.

Vervuert I, Coenen M, Bothe C. Effects of corn processing on the glycaemic and insulinaemic responses in horses. Journal of Animal Physiology and Animal Nutrition. 2004; 88(910):348-355. doi:10.1111/j.1439-0396.2004.00491.x.

Vervuert I, Voigt K, Hollands T, Cuddeford D, Coenen M. Effect of feeding increasing quantities of starch on glycaemic and insulinaemic responses in healthy horses. The Veterinary Journal. 2009; 182(1):67-72. doi:10.1016/j. tvjl.2008.04.011.

Recebido em: 11/08/2015

Received in: 08/11/2015

Aprovado em: 07/12/2015

Approved in: 12/07/2015 